\title{
Ex utero Intrapartum Treatment Procedure for Fetal Neck Teratoma
}

\author{
${ }^{1}$ Rula Al-Baghdadi, ${ }^{2}$ Nourhan Kika, ${ }^{3}$ Badreldeen Ahmed, ${ }^{4}$ Selma Porovic
}

\section{ABSTRACT}

A 24-year-old woman, G5P4, presented for a routine checkup at 28 weeks gestation. Ultrasound of the fetus showed a neck mass with no other anomalies and no polyhydramnios. Magnetic resonance imaging (MRI) of the fetus showed a cystic mass suggestive of a neck teratoma. She was continued with her pregnancy as planned and was scheduled for a cesarean section at 37 weeks gestation. Ex utero intrapartum treatment (EXIT) procedure was performed. There were no intraoperative or postoperative complications.

Keywords: Airway obstruction, Exit procedure, Fetal neck teratom.

How to cite this article: Al-Baghdadi R, Kika N, Ahmed B, Porovic S. Ex utero Intrapartum Treatment Procedure for Fetal Neck Teratoma. Donald School J Ultrasound Obstet Gynecol 2017;11(3):227-229.

Source of support: Nil

Conflict of interest: None

\section{HISTORY}

Mrs RM is a 24-year-old woman, G5P4, who was at 28 weeks gestation when she presented for a routine checkup. She had no complications up until that point in her pregnancy. Her past four pregnancies were uncomplicated and she delivered her children through normal vaginal deliveries. Prior to conception, her menstruations were regular. Her past medical and surgical histories are noncontributory. She does not have any allergies and is not on any medications.

\section{PHYSICAL EXAMINATION}

Physical examination was unremarkable. There was no pitting edema or signs of polyhydramnios.

\footnotetext{
1,2 Medical Student, ${ }^{3}$ Professor and Director, ${ }^{4}$ Director

1,2Department of Obstetrics and Gynecology, Weill Cornell Medicine, Doha, Qatar

${ }^{3}$ Department of Obstetrics and Gynecology, Weill Cornell Medicine, Feto-Maternal Medical Center, Doha, Qatar

${ }^{4}$ Department of Preventive and Pediatric Dentistry, Public Health Center of Sarajevo Canton, Sarajevo, Bosnia and Herzegovina

Corresponding Author: Badreldeen Ahmed, Professor and Director, Department of Obstetrics and Gynecology, Weill Cornell Medicine, Feto-Maternal Medical Center, Doha, Qatar, e-mail: profbadreldeen@hotmail.com
}

\section{ULTRASOUND FINDINGS}

The ultrasound showed that the fetus had a neck mass (Fig. 1) but had no other obvious anomalies. The mass was partially cystic and partially solid. Doppler ultrasound showed normal flow within the mass with normal resistance. The stomach was visualized, indicating no esophageal obstruction. The spine was visualized in coronal, sagittal, and axial views and it was intact. The rest of the fetal anatomy was normal. Fetal echo was done and it was normal. There was no polyhydramnios. Karyotyping was judged to be unnecessary.

\section{MAGNETIC RESONANCE IMAGING FINDINGS}

The MRI showed that the fetus had a large cystic neck mass (Fig. 2) that was highly suggestive of a neck teratoma.

\section{MANAGEMENT}

Mrs RM continued with her pregnancy as planned with repeated ultrasounds to monitor the growth of the teratoma and was scheduled for a cesarean section at 37 weeks of gestation. In view of her condition, the patient was scheduled for an EXIT procedure. To prepare for this procedure, we coordinated a team of fetal medicine specialist, pediatric surgeon, pediatric ear, nose, and throat (ENT) specialist, and neonatologists. The fetus was partially delivered with only the head and arms, and it was then intubated while the umbilical cord was still attached. Once airway was secured, the fetus

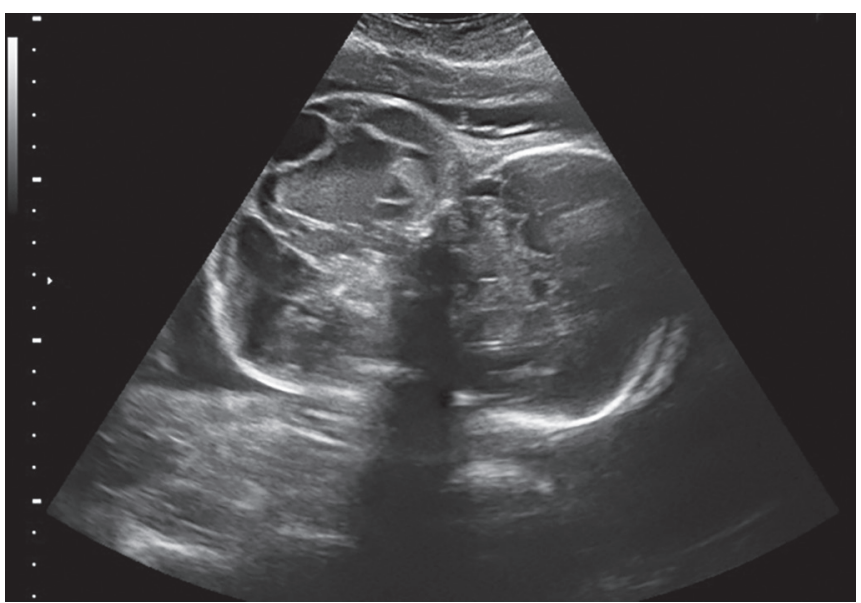

Fig. 1: Ultrasound image of the fetus with the neck mass and no other anomalies 


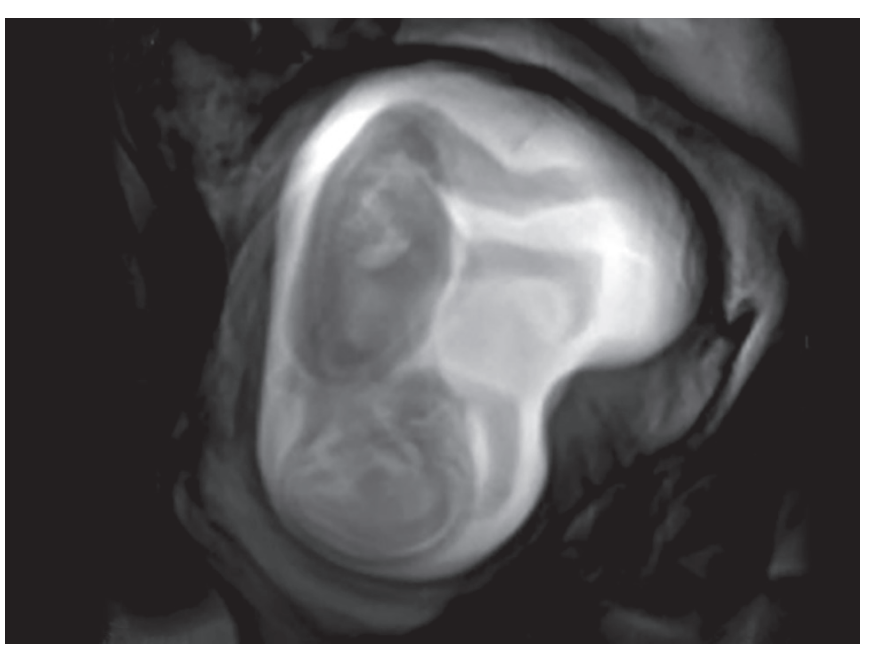

Fig. 2: Magnetic resonance imaging of the fetus with a large cystic neck mass

was completely delivered and the umbilical cord was detached. The surgery was uneventful.

\section{DISCUSSION}

A teratoma is a rare congenital tumor that can occur in the sacrum and coccyx, mediastinum, abdomen, head, or neck. These tumors are known to be fast-growing tumors and can cause compression symptoms depending on its location. ${ }^{1}$ Although it is a benign tumor, there is an increased risk of hydrops fetalis, premature delivery due to the size of the tumor, and airway obstruction if it occurs in the neck. ${ }^{2}$ Those located in the abdomen have the best prognosis. ${ }^{1}$

The fourth most common site of teratomas is in the head and neck. They usually present with polyhydramnios due to impaired fetal swallowing and the diagnosis is confirmed through ultrasound. The common complication with neck teratomas is airway obstruction. Ex utero intrapartum treatment (Figs 3 and 4) procedure has been shown to improve mortality and intubation is usually successful and a tracheostomy is usually not needed.

'Prenatal diagnosis of teratomas has been made possible through routine ultrasounds. Ultrasound imaging would typically show misalignment of the head and neck with the head in an opisthotonic position. ${ }^{3}$ Magnetic resonance imaging is used once a diagnosis is made to show more accurately the relationship of the tumor with the surrounding anatomy. ${ }^{4}$

The EXIT procedure is utilized in cases where the fetus's airway may be compromised. The aim of this procedure is to maintain the fetus on placental circulation after the partial delivery of the fetus. During this time, an airway is secured through endotracheal intubation, bronchoscopy, laryngoscopy, or excision of the mass. ${ }^{5}$ Uteroplacental circulation is maintained through uterine

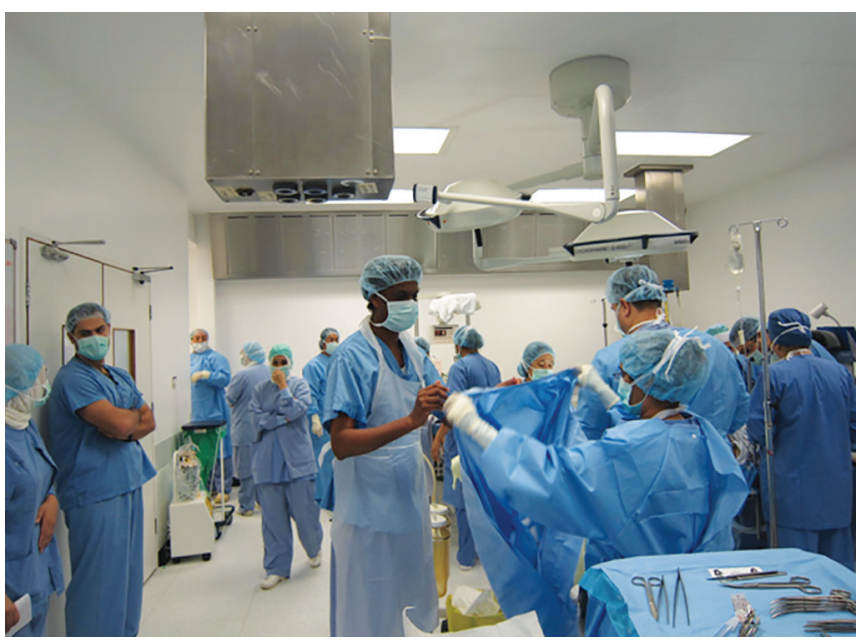

Fig. 3: Preoperation preparation involving the fetal medicine specialist, pediatric surgeon, pediatric ENT specialist, and neonatologists

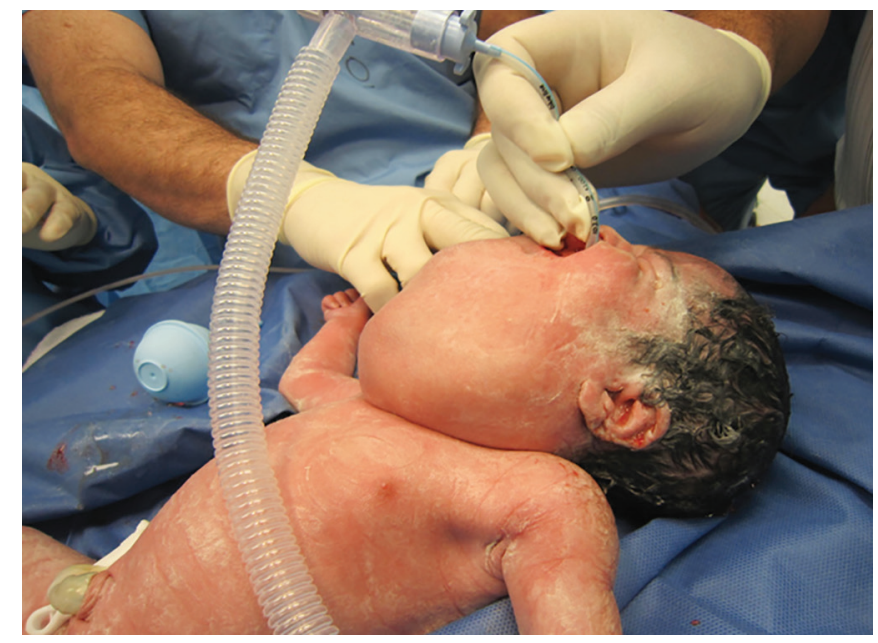

Fig 4: Neonate postintubation and cesarean section

relaxation by deep inhalation anesthesia and maintenance of uterine volume by only partially delivering the fetus. ${ }^{6}$

The EXIT procedure carries the risk of complications, the most serious of which is uterine atony. Uterine atony can occur due to the usage of high concentrations of deep inhalation anesthesia. This increases the risk of maternal hemorrhage and in severe cases could lead to hysterectomy. Placental injury can occur if the borders of the placenta are not accurately visualized through imaging prior to the procedure. Uterine rupture can occur in future pregnancies after the EXIT procedure. This occurs if the classical uterine incision is used. Ideally, a low anterior transverse uterine incision is done to prevent this future complication, but due to the position of the placenta, that cannot always be guaranteed. There is also a risk of fetal complications if uteroplacental circulation is compromised during the procedure due to cord compression, placental abruption, or loss of uterine relaxation. 
A cesarean section with EXIT procedure is planned in cases of fetal neck teratomas to avoid complications, such as dystocia, airway obstruction, and tumor avulsion. ${ }^{7}$ Planning the procedure involves coordination among different fields including obstetricians, neonatologists, pediatric surgeons, pediatric anesthesiologists, and pediatric otolaryngologists. ${ }^{8}$

Outcomes reported due to the EXIT procedure are limited due to the small number of facilities performing the procedure. One of the largest studies done regarding the outcome of those who underwent the EXIT procedure showed a $5 \%$ mortality rate for airway cases and a $25 \%$ mortality for complex airways. ${ }^{9}$ Another study showed that out of 31 women who underwent the EXIT procedure, only one fetus was lost during the procedure. ${ }^{10}$ The EXIT procedure has transformed the management of fetal airway obstruction; congenital malformations and tumors that were previously thought to be fatal can now be delivered through this procedure. ${ }^{9}$

\section{REFERENCES}

1. Peiró JL, Sbragia L, Scorletti F, Lim FY, Shaaban A. Management of fetal teratomas. Pediatr Surg Int 2016 July;32(7):635-647.
2. BrodskyJR,IraceAL,DidasA, WattersK, EstroffJA,BarnewoltCE, Perez-Atayde A, Rahbar R. Teratoma of the neonatal head and neck: a 41-year experience. Int $J$ Pediatr Otorhinolaryngol 2017 Jun;97:66-71.

3. Butler CR, Maughan EF, Pandya P, Hewitt R. Ex utero intrapartum treatment (EXIT) for upper airway obstruction. Curr Opin Otolaryngol Head Neck Surg 2017 Apr;25(2):119-126.

4. Thawani JP, Randazzo MJ, Singh N, Pisapia JM, Abdullah KG, Storm PB. Management of giant cervical teratoma with intracranial extension diagnosed in utero. J Neurol Surg Rep 2016 Jul;77:e118-e120.

5. Özgünen FT, Güleç UK, Evrüke IC, Demir SC, Büyükkurt S, Yapıcıoğlu H, İskit S. Fetal oropharyngeal and neck tumors: determination of the need for ex-utero intrapartum treatment procedure. Balkan Med J AVES 2015 Apr;32(2):221-225.

6. Liechty KW. Ex-utero intrapartum therapy. Semin Fetal Neonatal Med 2010 Feb;15(1):34-39.

7. CansaranS, Celayir AC, MoralığluS, Ayvacı H, TuğrulS, OvalıF, Çetiner H. The EXIT for prenatally diagnosed cervical cystic teratoma: a case report. J Neonatal Surg 2015 Apr-Jun;4(2):18.

8. Hirose $S$, Harrison MR. The ex utero intrapartum treatment (EXIT) procedure. Semin Neonatol 2003 Jun;8(3):207-214.

9. Butler CR, Maughan EF, Pandya P, Hewitt R. Ex utero intrapartum treatment (EXIT) for upper airway obstruction. Curr Opin Otolaryngol Head Neck Surg 2017 Apr;25(2):119-126.

10. Abraham RJ, Sau A, Maxwell D. A review of the EXIT (ex utero intrapartum treatment) procedure. J Obstet Gynaecol 2010 Jan;30(1):1-5. 